\title{
Separating Thickness from Geometric Thickness
}

\author{
David Eppstein \\ Dept. of Information and Computer Science \\ University of California, Irvine \\ Irvine, CA 92697-3425, USA \\ eppstein@ics.uci.edu
}

\begin{abstract}
We show that graph-theoretic thickness and geometric thickness are not asymptotically equivalent: for every $t$, there exists a graph with thickness three and geometric thickness $\geq t$.
\end{abstract}

\section{Introduction}

The graph-theoretic property of thickness and its variants formalize the concept of layered graph drawing, in which a graph must be drawn in multiple layers (or with multiple colors) so that each edge belongs to a single layer and no two edges in the same layer cross. The vertices of the graph should be represented as points and should exist in the same positions in each layer. There are three important variants of thickness:

- The thickness of a graph $G$, denoted $\theta(G)$, is the minimum number of layers for which a drawing of $G$ exists, without restriction on the number of bends per edge [11.

- The geometric thickness of a graph $G$, denoted $\bar{\theta}(G)$, also known as real linear thickness, is the minimum number of layers for which a drawing exists without any bends in the edges: each edge must be represented as a straight line segment [5, 11].

- The book thickness of a graph $G$, denoted bt $(G)$, is the minimum number of layers for which a drawing exists without any bends in the edges and with all vertices placed in convex position [4].

Since each variant imposes additional constraints on the previous one, these parameters are always ordered: $\theta(G) \leq \bar{\theta}(G) \leq \mathrm{bt}(G)$, and it is of interest to determine how tight these inequalities are. Due to Fáry's theorem [7], the graphs with thickness one and geometric thickness one coincide (both are just the planar graphs), and are a strict superset of the graphs with book thickness one (the outerplanar graphs). Thickness and geometric thickness were known to differ for infinitely many graphs: in particular, complete graphs on $n$ vertices have thickness $n / 6+O(1)[1,2,3,13,14$, but have geometric thickness $\geq n / 5.646$ [5]. In previous work [6] we showed that book thickness can not be bounded by any function of the geometric thickness: for any $t$ there exists a graph with geometric 


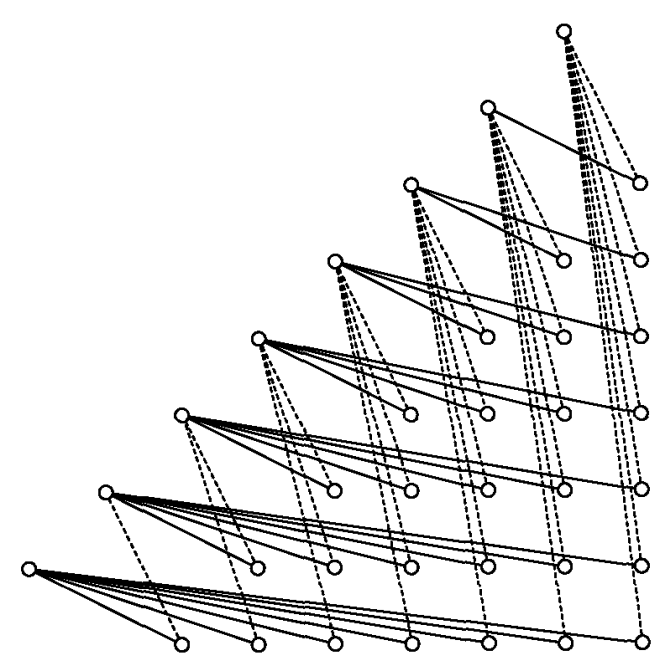

Fig. 1. Graph $G_{2}(8)$ drawn with geometric thickness two.

thickness two and book thickness $\geq t$. Therefore, one can not hope to find nearoptimal bend-free layered drawings by placing all vertices in convex position.

In this paper, we prove a similar separation between thickness and geometric thickness: for any $t$ there exists a graph with thickness three and geometric thickness $\geq t$. This implies that known strategies for approximating thickness (layered drawing with bends) will not lead to good approximation algorithms for layered drawing without bends.

\section{Proof Outline}

The overall outline of our proof is very similar to our previous result, separating geometric thickness from book thickness, so as a warmup we review that result.

Theorem 1 (Eppstein [6]). For every t, a graph exists with geometric thickness two and book thickness $\geq t$.

Proof. For any $n>0$, define a graph $G_{2}(n)$ having as its $n+\left(\begin{array}{c}n \\ 2\end{array}\right)$ vertices the singleton and doubleton subsets of an $n$-element set, with an edge between two subsets when one is contained in the other. $G_{2}(n)$ can be drawn with geometric thickness two using a construction related to a one-bend drawing algorithm of Wood [15]; a two-layer drawing of $G_{2}(8)$ is shown in Figure 1. However, $G_{2}(5)$ is nonplanar (it is formed by subdividing each edge of the nonplanar complete graph $K_{5}$ ) so it has book thickness greater than two.

By Ramsey theory (Lemma 1), there exists an $n_{0}$ such that, if the edges of $G_{2}\left(n_{0}\right)$ are given $t-1$ colors, one can find a two-colored $G_{2}(5)$ subgraph. If the $(t-1)$-coloring is determined by a book embedding with $t-1$ layers, the subgraph would have a two-layer book embedding. Since no such two-layer embedding exists, $G_{2}\left(n_{0}\right)$ does not have a $(t-1)$-layer book embedding. 


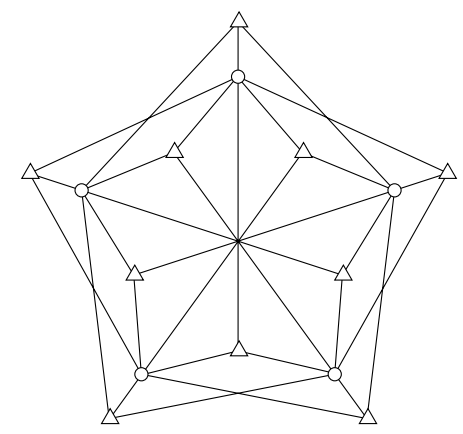

Fig. 2. Graph $G_{3}(5)$. The circular vertices represent singleton subsets, and the triangular vertices represent tripleton subsets.

Our main result is the following theorem.

Theorem 2. For every $t$, a graph exists with thickness three and geometric thickness $\geq t$.

Proof. For any $n>0$, define a graph $G_{3}(n)$ having as its $n+\left(\begin{array}{l}n \\ 3\end{array}\right)$ vertices the singleton and tripleton subsets of an $n$-element set, with an edge between two subsets when one is contained in the other. $G_{3}(4)$ forms the vertices and edges of a cube; $G_{3}(5)$ is depicted in Figure2. If we assign the edges of $G_{3}(n)$ to three layers, in such a way that the three edges incident to each tripleton are assigned to different layers, then each layer will form a forest of stars, which is a planar graph, so $G_{3}(n)$ has thickness at most three. We show that there exists an $n_{1}$ such that $G_{3}\left(n_{1}\right)$ has geometric thickness more than three (Lemma 19).

By Ramsey theory (Lemma 1), there exists $n_{2}$ such that, if the edges of $G_{3}\left(n_{2}\right)$ are given $t-1$ colors, it has a three-colored $G_{3}\left(n_{1}\right)$ subgraph. If the $(t-1)$-coloring is determined by a $(t-1)$-layer geometric embedding, the subgraph would have a three-layer geometric embedding. Since no such three-layer embedding exists, $G_{3}\left(n_{2}\right)$ does not have a $(t-1)$-layer geometric embedding.

The difficult part of our proof is Lemma 19, showing that some $G_{3}(n)$ has geometric thickness greater than three. The proof involves a complicated case analysis, with Ramsey theory used again at several stages in order to show that, if a thickness-three drawing existed, one could find a drawing with a simplified form that can be shown not to exist.

\section{Ramsey Theory}

We use the following classical result from Ramsey theory [?, Theorem 2]:

Lemma 1. For every three positive integers $c$, $e$, and $\ell$ there is an integer $R_{e}(\ell ; c)$ with the following property: if $|S| \geq R_{e}(\ell ; c)$ and the e-element subsets of $S$ are partitioned into $c$ classes, then there exists a set $T \subset S$ with $|T| \geq \ell$ so that all e-element subsets of $T$ belong to the same class. 
For instance, in the proof of Theorem 1, we choose $c=\left(\begin{array}{c}t-1 \\ 2\end{array}\right)+t-1, e=2$, and $\ell=5$. The set $S$ is the set of singletons in $G_{2}\left(n_{0}\right)$, and the doubletons in $G_{2}\left(n_{0}\right)$ are partitioned into $c$ classes by the colors of their two incident edges. The Lemma tells us that we can find a two-colored $G_{2}(5)$ subgraph of $G_{2}\left(n_{0}\right)$. Similarly, in Theorem 2 we can find a three-colored $G_{3}\left(n_{1}\right)$ subgraph of $G_{3}\left(n_{2}\right)$.

We also use another well-known Ramsey-theoretic result [?, pp. 24-25]:

Lemma 2. For every integer $k$ there is an integer $N(k)$ with the following property: if $N(k)$ points are placed in the plane with no three points colinear, then some subset of $k$ points form the vertices of a convex polygon.

\section{Coherent Drawings}

Define a convex drawing of $G_{3}(n)$ to be a drawing with geometric thickness three in which all singletons are in convex position. We do not restrict the positions of the tripletons.

Lemma 3. If every graph $G_{3}(n)$ has geometric thickness three, then every $G_{3}(n)$ has a convex drawing.

Proof. Small perturbations of the vertex locations in a layered drawing will not create or remove crossings, so we can assume no three vertices are colinear. To form a convex drawing of $G_{3}(n)$, apply Lemma 2 to the singletons of $G_{3}(N(n)$ ), and form a $G_{3}(n)$ subgraph from the resulting convex set of $n$ singletons and their adjacent tripletons.

We number the singleton vertices of a convex drawing in clockwise order from 0 to $n-1$, starting from an arbitrarily chosen vertex. Using this numbering, we can partition the edges of $G_{3}(n)$ into three classes: a low edge connects a tripleton to the adjacent singleton with the smallest number, a high edge connects a tripleton to the adjacent singleton with the largest number, and a middle edge connects a tripleton to the remaining adjacent singleton. Define a coherent drawing of $G_{3}(n)$ to be a convex drawing in which these three classes form the three layers of the drawing.

Lemma 4. If every graph $G_{3}(n)$ has geometric thickness three, then every $G_{3}(n)$ has a coherent drawing.

Proof. By Lemma 3 we can assume $G_{3}(n)$ has a convex drawing. Partition the tripletons of such a drawing into 27 classes according to the layers to which the incident low, middle, and high edges belong. By Lemma 1 with $c=27$ and $e=3$, we can find a drawing in which each of the three sets of edges belongs to a single layer. If this results in more than one set belonging to the same layer, we can move sets to distinct layers without introducing any crossings.

In a coherent drawing, we will denote tripletons by triples of symbols $a b c$, where each letter denotes the position of an adjacent singleton in the clockwise ordering of the drawing, and $a<b<c$ according to the clockwise ordering. 

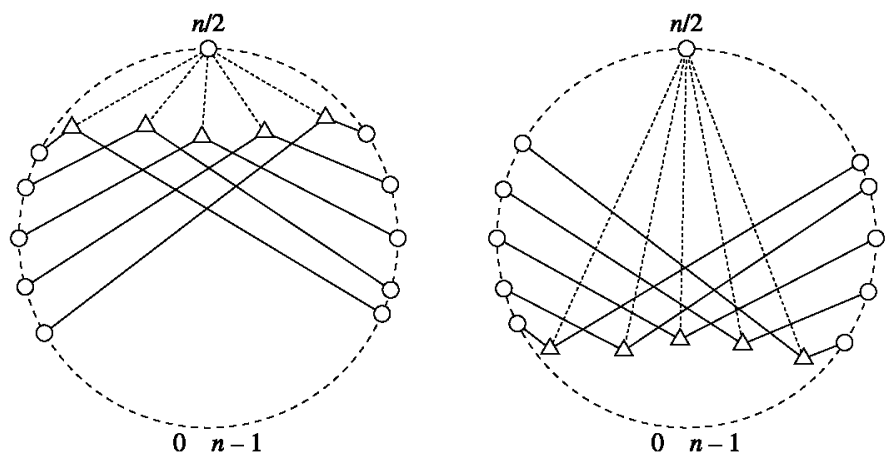

Fig. 3. Five tripletons crossing each other convexly (left) or concavely (right), from Lemma 6.

\section{$5 \quad$ Restriction to Outer Drawings}

Define an inner drawing of $G_{3}(n)$ to be a coherent drawing such that some strictly convex curve $C$ passes through all singletons and contains all tripletons. Similarly, define an outer drawing to be a coherent drawing such that some strictly convex curve $C$ passes through all singletons and contains no tripletons. A drawing can be both inner and outer, for instance if all vertices are in convex position. In an inner drawing, all edges are contained in $C$; however an edge in an outer drawing may have portions inside $C$ and portions outside $C$.

Lemma 5. Suppose two tripletons abc and def are part of an inner drawing, with $a<d<c<f$. Then either the low edge of abc crosses the high edge of def, or the high edge of abc crosses the low edge of def. However, both types of crossing can not occur simultaneously.

Proof. Consider the two paths formed by the low and high edges of each tripleton. These paths cross $C$ and each path separates the endpoints of the other path; therefore the paths cross an odd number of times. However, each pair of edges can cross only once, and the only types of crossing permitted are those described in the lemma, so exactly one of these crossings occurs.

We say that the two tripletons cross convexly if the low edge of $a b c$ crosses the high edge of def, and that they cross concavely in the other case of Lemma 5 .

Lemma 6. There exists an $n_{3}$ such that $G_{3}\left(n_{3}\right)$ does not have an inner drawing.

Proof. Let $n$ be sufficiently large, and assume for convenience that $n=2$ $(\bmod 4)$. Consider the sequence of tripletons $2 i, n / 2, n / 2+2 i+2$ for $0 \leq i<$ $n / 2-2$; each pair of tripletons meets the conditions of Lemma 5 . By applying Lemma 1 with $c=2, e=2$, and $\ell=5$, for sufficiently large $n$, we can obtain a set of five such triples that all cross convexly or all cross concavely. As Figure 3 shows, in either case, the low and high edges of these crossing triples form a grid 

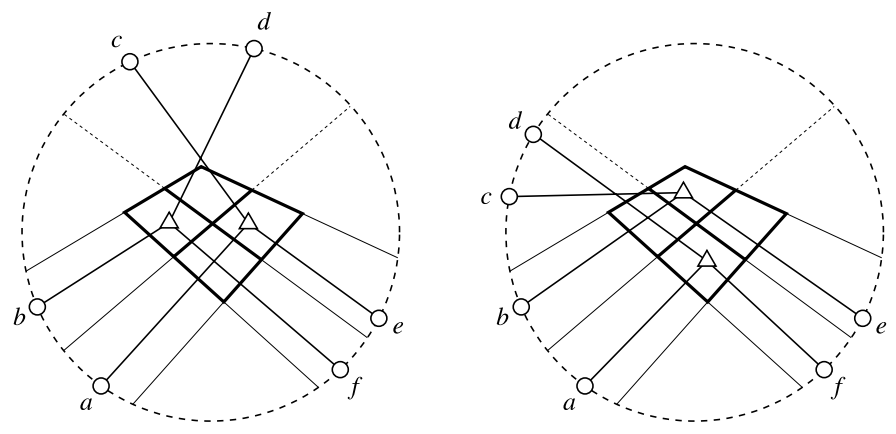

Fig. 4. Forced crossings in an inner drawing, from Lemma 6.

graph, containing a complete $2 \times 2$ subgrid. (The quadrilaterals of this grid need not always be convex, but this does not affect our argument.)

Because we chose tripletons using only every other singleton from the ordering, it is now possible to find singletons $a$ and $b$ that lie between the three low edges forming the $2 \times 2$ subgrid, and $e$ and $f$ that lie between the three high edges forming the subgrid (Figure 4). Therefore, any tripleton with a low edge to $a$ or $b$ and a high edge to $e$ or $f$ must be forced to lie within a particular square of the subgrid.

Extend the two middle line segments of the subgrid into lines across curve $C$ (the thin dotted lines of the figure), partitioning $C$ into three curves: the curve clockwise of both extended lines, the curve counterclockwise of the extended lines, and the curve between the two lines. The four points $n / 2-2, n / 2-1, n / 2$, $n / 2+1$ lie between $b$ and $e$, and by the pigeonhole principle we can find two of these points $c<d$ that are both within the same one of these three curves.

If $c$ and $d$ are both in the center curve of the three curves (shown in the left side of the figure) then tripletons ace and bdf must have crossing middle edges. If $c$ and $d$ are both in the counterclockwise curve (shown on the right side of the figure), then tripletons $a d f$ and bce must have crossing middle edges; the case in which $c$ and $d$ belong to the clockwise curve is symmetric. Thus, in all cases two tripletons have crossing middle edges, contradicting the assumption that we have a three-layer drawing with no crossings.

Lemma 7. If every graph $G_{3}(n)$ has geometric thickness three, then every $G_{3}(n)$ has an outer drawing.

Proof. Assume without loss of generality that $n$ is sufficiently large that, by Lemma 6 , no inner drawing of $G_{3}(n)$ exists. Let $r=R_{3}(n ; 2)$, as shown to exist in Lemma 1 By Lemma 4, we can assume $G_{3}(r)$ has a coherent drawing. Let $C$ be the convex hull of the singletons of this drawing, and perturb $C$ if necessary so that it remains convex and does not pass through any tripletons. Partition the tripletons of the drawing into two classes according to whether they are inside or outside $C$, and apply Lemma 1 with $c=2$ and $e=3$ to find a drawing 

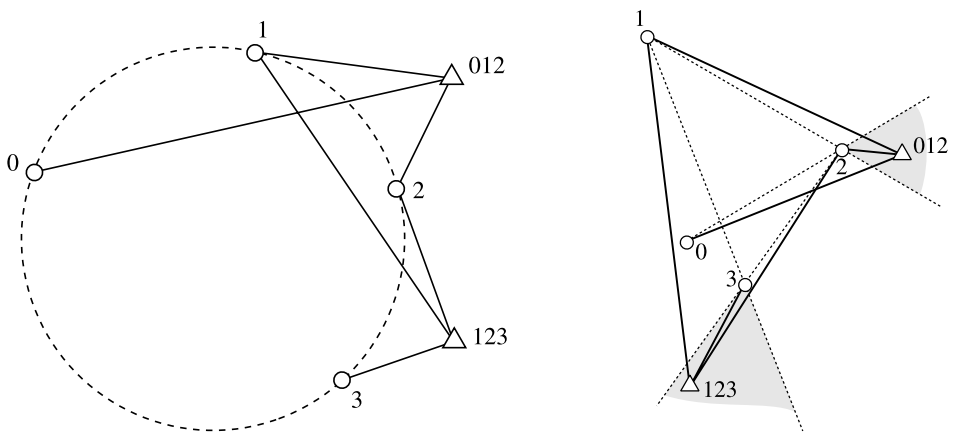

Fig. 5. Left: Forced crossing in an outer drawing of type 201, from Lemma 9] Right: Sharp angles in an outer drawing of type 021, from Lemma 10.

of $G_{3}(n)$ in which all triples are inside $C$ or all triples are outside $C$. Since no inner drawing exists, all triples are outside $C$, and we have an outer drawing of $G_{3}(n)$.

\section{Classification of Outer Drawings}

In an outer drawing, the three angles formed by the three edges incident to a tripleton must include an angle greater than $180^{\circ}$, and we can order the three edges clockwise starting at this large angle. Define the type of a tripleton to be a symbol $x y z$, where $x, y$, and $z$ form a permutation of 0,1 , and 2 , and where the positions of the low, middle, and high number in the symbol are the same as the positions of the low, middle, and high edge in the clockwise ordering at the tripleton. There are six possible types: $012,021,102,120,201$, and 210 . If all tripletons in a drawing have the same type $x y z$, we say that the drawing is of type $x y z$.

Lemma 8. If every graph $G_{3}(n)$ has geometric thickness three, then every $G_{3}(n)$ has an outer drawing in which all tripletons have the same type.

Proof. By the previous lemma, we can assume that we have an outer drawing with sufficiently many vertices, to which we can apply Lemma 1 with $c=6$ and $e=3$.

We now successively analyze each type of outer drawing and show that, for sufficiently large $n$, each type must lead to a crossing.

Lemma 9. For $n \geq 4, G_{3}(n)$ has no outer drawing of type 201 or 120.

Proof. In a drawing of type 201, each low edge of a tripleton must cross the convex curve $C$ between the middle and high sigletons adjacent to the tripleton. Therefore, in a drawing of $G_{3}(4)$, the low edge from tripleton 012 would cross the low edge from tripleton 123, as shown in Figure 5. Type 120 is equivalent 
to type 201 under mirror reversal of the drawing and reversal of the clockwise ordering of the singletons.

Lemma 10. In any outer drawing of $G_{3}(4)$ of type 021 , let $\theta_{i}$ denote the angle at singleton $i$ on the convex hull of the singletons. Then $\theta_{1}+\theta_{2}<180^{\circ}$.

Proof. In order to be of type 021, tripleton 012 must lie in a wedge outside $C$ bounded by lines 02 and 12 (the shaded area on the upper right of Figure 5). The low edge $e_{0}$ from 0 to 012 crosses line 12 on the boundary of this wedge. Similarly, the low edge $e_{1}$ from 1 to tripleton 123 crosses line 23. In order for $e_{1}$ to reach this line without crossing $e_{0}$, tripleton 123 must be clockwise from singleton 0 as viewed from singleton 1, so angle 123-1-2 must be greater than $\theta_{1}$. But in order for the crossing of $e_{1}$ with line 23 to be on the boundary of the wedge containing 123 (shown as the lower shaded area in the figure), angle 123-1-2 must be less than $180^{\circ}-\theta_{2}$. Combining these two inequalities gives the result.

Lemma 11. For $n \geq 6, G_{3}(n)$ has no outer drawing of type 021 or 102.

Proof. By applying Lemma 10 twice, $\theta_{1}+\theta_{2}+\theta_{3}+\theta_{4}<360^{\circ}$, but this is not possible in a convex polygon. Type 102 is equivalent to type 021 under mirror reversal.

Lemma 12. In an outer drawing of $G_{3}(n)$ of type 012 , the middle edges adjacent to singletons $2,3, \ldots, n-3$ all pass across line segment $0-(n-1)$.

Proof. By the assumption that the drawing is of type 012, the middle edges of the tripletons $0 i(n-1)$ all pass across this segment (Figure 6. left). In any other singleton $a b c$, the middle edge must pass from $b$ across line segment $a c$ within the convex curve $C$. Unless $b=1$ or $b=n-2$, this crossing with $a c$ must occur within a strip bounded by two of the middle edges of tripletons $0 i(n-1)$, and the only way for the middle edge from $b$ to exit $C$ is at the end of this strip, where it meets segment $0-(n-1)$.

Lemma 13. Suppose we have an outer drawing of $G_{3}(n)$ of type 012 , in which all middle edges cross segment $0-(n-1)$. Draw a tangent line to convex curve $C$ parallel to line $0-(n-1)$, on the opposite side of $C$ from that line, and let $h$ be a point where the tangent line touches $C$. Then, for every tripleton ijk where $i$ is clockwise from $h$, the low edge from $i$ to ijk crosses $C$ either at a point counterclockwise from $h$ or between singletons 0 and $n-1$.

Proof. By the assumption of the drawing type, all low edges must cross line 0$n-1$ to the left of the crossing point of the middle edge, which is between points 0 and $n-1$. If the low edge's crossing is not also between points 0 and $n-1$, it must be to the left of point 0 . But, within the strip between line $0-(n-1)$ and the parallel tangent line, the portion of $C$ between 0 and $h$ separates the rest of $C$ (containing $i$ ) from the points left of point 0 (as shown in Figure 6, right), so the low edge most cross $C$ before it reaches its crossing with line $0-(n-1)$. 

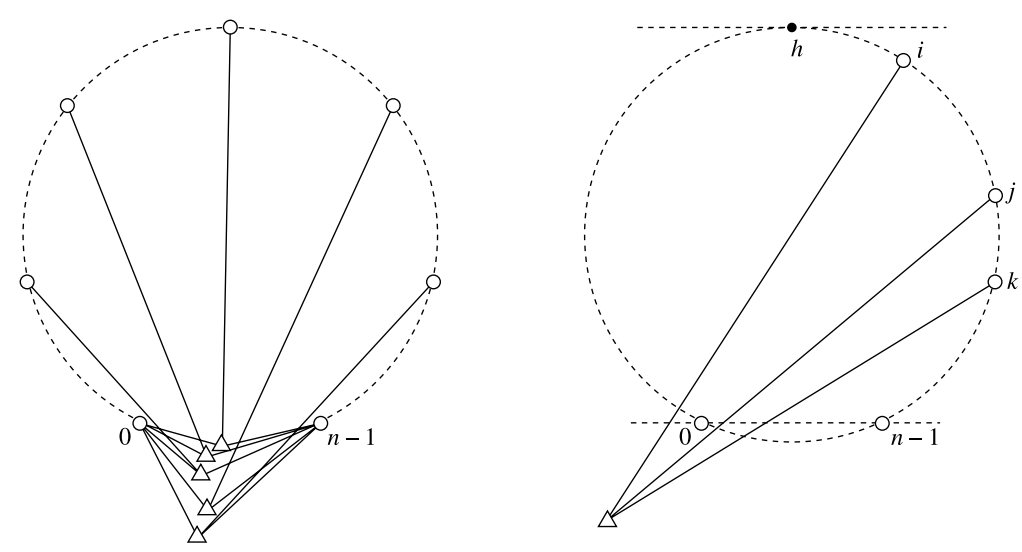

Fig. 6. Analysis of outer drawings of type 012. Left, the middle edges of the tripletons $0 i(n-1)$ form tracks that force the remaining middle edges to cross segment 0 - $(n-1)$ (Lemma 12). Right, low edges from clockwise of the tangent point $h$ cross $C$ counterclockwise of the tangent point (Lemma 13).

Symmetrically, the high edges from counterclockwise of $h$ must cross $C$ clockwise of $h$.

Lemma 14. For sufficiently large $n, G_{3}(n)$ has no outer drawing of type 012.

Proof. Start with an outer drawing of type 012, and remove singletons 1 and $n-2$ if necessary so that (by Lemma 12) all middle edges cross segment $0-(n-1)$. Form the tangent point $h$ described in Lemma 13, and assume without loss of generality that at least half of the remaining singletons lie clockwise of $h$ on convex curve $C$. (The case that at least half the singletons lie counterclockwise of $h$ is symmetric.) Remove from the drawing all points counterclockwise of $h$. The result is an outer drawing of type 012 of a graph $G_{3}(m)$, with at least $m \geq(n-2) / 2$ singletons, in which all low and middle edges cross segment 0 $(m-1)$. We can now apply the same reasoning as in Lemma 6: by choosing an appropriate set of tripletons, we can find a set of low and middle edges forming a grid that contains a complete $2 \times 2$ subgrid, and use the subgrid to constrain the locations of two more tripletons, forcing two high edges to cross.

Lemma 15. In an outer drawing of $G_{3}(n)$ of type 210, each middle edge is completely exterior to convex curve $C$.

Proof. Suppose to the contrary that a middle edge for tripleton $i j k$ crosses $C$ at point $x$. If $x$ is clockwise of $j$, an edge from $i j k$ to $C$ clockwise of the middle edge can only reach singletons in the range from $j$ to $x$, contradicting the requirement (from the assumption of the drawing type) that the low edge $i$-ijk must be clockwise of the middle edge. Symmetrically, if $x$ is counterclockwise of $j$, an edge counterclockwise of the middle edge can only reach singletons in the range 


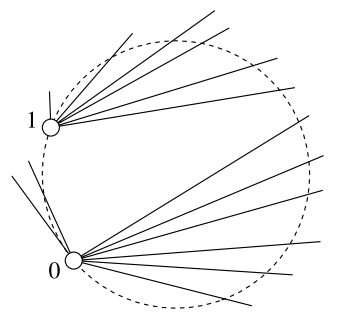

Fig. 7. In an outer drawing of type 210, low edges from singletons 0 and 1 cross disjoint ranges of the convex curve $C$ (Lemma 17 ).

from $x$ to $j$, contradicting the requirement that the high edge must be clockwise of the middle edge.

The same reasoning as above also proves the following lemma:

Lemma 16. In an outer drawing of $G_{3}(n)$ of type 210 , suppose that the low edge of tripleton ijk crosses the convex curve $C$. Then the crossing point is clockwise of singleton $i$.

Lemma 17. Suppose that $G_{3}(n)$ has a convex drawing of type 210 , and let $n=$ $2^{k}-2$ Then we can select a subset $S$ of the singletons, with $|S|=k$, such that no member of $S$ is incident to a low edge that crosses the convex hull of $S$.

Proof. We use induction on $k$. As a base case, for $k=n=2, G_{3}(2)$ has no edges. Otherwise, consider the sets of low edges incident to singletons 0 and 1 that cross convex curve $C$. Since these two sets of low edges do not cross each other (Figure 17), we can partition the portion of $C$ clockwise of 1 into two parts, where the clockwise part of $C$ is crossed only by low edges from 0 and the counterclockwise part of $C$ is crossed only by low edges from 1 . One of these two parts contains at least $(n-2) / 2$ singletons. If the clockwise part contains many singletons, we apply the induction hypothesis to these singletons, and form $S$ by adding singleton 1 to the resulting set. On the other hand, if the counterclockwise part contains many singletons, we add singleton 0 to the set formed by applying the induction hypothesis to the singletons in this part.

In either case, we get a set $S$ of $(k-1)+1=k$ singletons. The low edges from 0 or 1 (whichever was included in the set) can not cross the hull, because of how we chose which part of $C$ to apply the induction hypothesis to. The low edges from the remaining members of $S$ can not cross the hull between 0 or 1 and the rest of $S$, by Lemma 16, and they can not cross the hull elsewhere by induction. Therefore $S$ fulfills the conditions of the lemma.

Lemma 18. Not every graph $G_{3}(n)$ has an outer drawing of type 210.

Proof. By Lemma [17 if $G_{3}(n)$ has such a drawing, then we can find a set of $k$ singletons, and a convex curve $C$ (the hull of this set), forming an outer drawing 


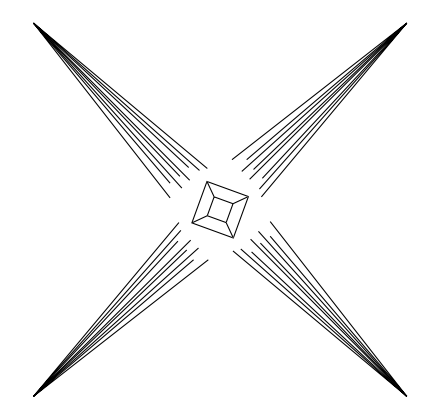

Fig. 8. Schematic view of drawing of $G_{3}(8)$ with geometric thickness three.

of $G_{3}(k)$ in which all low and middle edges avoid the interior of convex curve $C$; here $k=\left\lfloor\log _{2}(n+2)\right\rfloor$. We can now apply the same reasoning as in Lemmas 6 and 14 by choosing an appropriate set of tripletons, we can find a set of low and middle edges forming a grid that contains a complete $2 \times 2$ subgrid, and use the subgrid to constrain the locations of two more tripletons, forcing two high edges to cross.

\section{Completion of the Proof}

Lemma 19. Not every graph $G_{3}(n)$ has geometric thickness at most three.

Proof. This follows from Lemma 8 (showing that we can restrict our attention to outer drawings of fixed type) and Lemmas 9 11, 14, and 18 (showing that each fixed type leads to a forced crossing).

The largest $n$ for which $G_{3}(n)$ has no thickness-three drawing must be at least nine, as $G_{3}(8)$ has geometric thickness at most three: draw four singleton subsets in a large square, with the remaining four singletons and all tripletons clustered near the center of the square. The edges incident to the inner four singletons form a planar graph, consisting of a cube $G_{3}(4), 24$ two-edge paths across the faces of the cube, and 24 tripletons with only a single edge to one of the inner singletons. By choosing an appropriate Fáry embedding of this planar graph, we can draw it in a single layer, and use an additional layer for the edges incident to each pair of opposite outer singletons. Figure 8 shows this drawing in a schematic view, with the outer four vertices and inner cube visible but omitting the remaining tripletons.

\section{Conclusions}

We have shown that thickness and geometric thickness are not asymptotically equivalent. Some interesting open questions remain: 
- What can be said about two-layer drawings? It is known that there exist graphs, for instance $K_{6,8}$, for which $\theta(G)=2$ and $\bar{\theta}(G)>2$ [12, 5 . Can $\bar{\theta}(G)$ be unbounded when $\theta(G)=2$ ?

- Four-regular graphs have thickness at most two. Do random four-regular graphs have bounded expected geometric thickness? More generally, can one bound the geometric thickness of a graph as some function of its degree?

- Due to our use of Ramsey theory, our lower bounds on geometric thickness grow only very slowly as a function of the number of vertices. Can we find good upper bounds for geometric thickness as a combination of the graphtheoretic thickness and a slowly growing function of the graph size?

- What is the complexity of computing $\bar{\theta}(G)$ for a given graph $G[5]$ ?

- Thickness is NP-hard [12] but not difficult to approximate to within a constant factor; e.g., it is within a factor of three of the graph's arboricity. Our new results imply that these approximations do not directly extend to geometric thickness. Is there an efficient algorithm for layered drawing of graphs without bends, using a number of layers within a constant factor of optimal?

\section{Acknowledgements}

This work was supported in part by NSF grant CCR-9912338.

\section{References}

1. V. B. Alekseev and V. S. Gončakov. The thickness of an arbitrary complete graph. Math USSR Sbornik 30(2):187-202, 1976.

2. L. W. Beineke. The decomposition of complete graphs into planar subgraphs. Graph Theory and Theoretical Physics, chapter 4, pp. 139-153. Academic Press, 1967.

3. L. W. Beineke and F. Harary. The thickness of the complete graph. Canad. J. Math. 17:850-859, 1965.

4. F. Bernhart and P. C. Kainen. The book thickness of a graph. J. Combinatorial Theory, Ser. B 27(3):320-331, 1979.

5. M. B. Dillencourt, D. Eppstein, and D. S. Hirschberg. Geometric thickness of complete graphs. J. Graph Algorithms \& Applications 4(3):5-17, 2000, arXiv:math.CO/9910185.

6. D. Eppstein. Separating geometric thickness from book thickness. arXiv.org, September 2001, arXiv:math.CO/0109195.

7. I. Fáry. On straight line representation of planar graphs. Acta Sci. Math. Szeged. 11:229-233, 1948.

8. R. L. Graham, B. L. Rothschild, and J. H. Spencer. Ramsey Theory. Wiley, 1980.

9. N. Hartsfield and G. Ringel. Pearls in Graph Theory. Academic Press, Boston, MA, 1990.

10. B. Jackson and G. Ringel. Plane constructions for graphs, networks, and maps: Measurements of planarity. Selected Topics in Operations Research and Mathematical Economics: Proc. 8th Symp. Operations Research, pp. 315-324. Springer-Verlag, Lecture Notes in Economics and Mathematical Systems 226, August 1983.

11. P. C. Kainen. Thickness and coarseness of graphs. Abh. Math. Sem. Univ. Hamburg 39:88-95, 1973. 
12. A. Mansfield. Determining the thickness of a graph is NP-hard. Math. Proc. Cambridge Philos. Soc. 93(9):9-23, 1983.

13. J. Mayer. Decomposition de $K_{16}$ en trois graphes planaires. J. Comb. Th., Ser. B 13:71, 1972.

14. J. Vasak. The thickness of the complete graph having $6 m+4$ points. Manuscript, cited in [9] and [10.

15. D. R. Wood. Geometric thickness in a grid of linear area. Proc. Euroconf. Combinatorics, Graph Theory, and Applications, pp. 310-315. Univ. Autònoma de Barcelona, Centre de Recerca Matemàtica, September 2001, http://www.cs.usyd.edu.au/ davidw/papers/Wood-COMB01.ps. 